\title{
Risk of second cancer among young prostate cancer survivors
}

\author{
Hong Zhang ${ }^{1}$, Andrew $\mathrm{Yu}^{2}$, Andrea Baran ${ }^{3}$, Edward Messing ${ }^{4}$ \\ ${ }^{1}$ Department of Radiation Oncology, University of Rochester Medical Center, Rochester, NY, USA \\ ${ }^{2}$ Department of Neurology, Johns Hopkins School of Medicine, Baltimore, MD, USA \\ ${ }^{3}$ Department of Biostatistics and Computational Biology, University of Rochester Medical Center, Rochester, NY, USA \\ ${ }^{4}$ Department of Urology, University of Rochester Medical Center, Rochester, NY, USA
}

Received: October 18, 2020

Revised: April 5, 2021

Accepted: April 12, 2021

Correspondence:

Hong Zhang

Department of Radiation Oncology, University of Rochester Medical

Center, 601 Elmwood Ave, Box 647,

Rochester, NY 14642, USA

Tel: +1-585-341-6750

E-mail: hong_zhang@urmc.

rochester.edu

ORCID:

https://orcid.org/0000-0003-3631-9163
Purpose: About $40 \%$ of men diagnosed with prostate cancer (Pca) are $\leq 65$ years of age. This study evaluates the risk of second cancer among young Pca patients treated with surgery or radiation. Materials and Methods: This is a retrospective review of 150,915 men aged $\leq 65$ years at Pca diagnosis treated with surgery or radiation registered in the Surveillance, Epidemiology, and End Results (SEER) database between 1973 and 2014. Incidence rates of second rectum/rectosigmoid junction $(\mathrm{RJ})$, bladder, and lung cancer in each treatment group were reported with adjustment for potential confounders. Cumulative incidence functions were used to summarize the risk of second cancer after completing initial treatment.

Results: Men treated with external beam radiation (BEAM), brachytherapy (SEED), or combined radiation all exhibited a statistically significant increased incidence of second bladder cancer compared to men treated with surgery (adjusted incidence rate ratio [IRR]: 2.09, 1.91, and 2.04, respectively). Incidence of rectum/RJ cancer was also significantly increased in men receiving BEAM and combined radiation (adjusted IRR: 1.58 and 1.98, respectively). There were also significant differences in the cumulative incidence of second bladder cancer after receiving any form of radiation compared to surgery.

Conclusion: Pca survivors $\leq 65$ years of age at Pca diagnosis had an increased risk of second bladder and rectum/RJ cancer after BEAM and combined radiation treatment after adjusting for confounding factors. Second bladder cancer incidence after either form of radiation treatment was increased even at 5 years after a Pca diagnosis.

Keywords: Prostate cancer, Second cancer, Radiotherapy, Prostatectomy

\section{Introduction}

Prostate cancer is the second most common cancer in men, behind only skin cancer. There will be an estimated 191,930 new prostate cancer cases diagnosed in 2020; 40\% will occur in men $\leq 65$ years of age [1]. Not all men diagnosed with prostate cancer, however, will die from the disease. It has been estimated that 3.1 million men who have at some point been diagnosed with prostate cancer are alive today [2]. Prostate cancer survivors face many challenges, including the risk of a second primary cancer $[3,4]$. This challenge is more pronounced in younger men due to their longer post-diagnosis life expectancy.

Many factors may contribute to the risk of second primary cancer among prostate cancer survivors. Some are similar to those in men with no prior cancer diagnosis, such as lifestyle, environmental exposure, and genetic susceptibility. External beam radiation (BEAM), brachytherapy (permanent seed implants or high-doserate brachytherapy, SEED), or a combination of the two are used as less invasive alternatives to surgery for treating localized prostate cancer; radiation has been shown to increase the risk of bladder

Copyright (C) 2021 The Korean Society for Radiation Oncology

This is an Open Access article distributed under the terms of the Creative Commons Attribution Non-Commercial License (http://creativecommons.org/licenses/by$\mathrm{nc} / 4.0 /$ ) which permits unrestricted non-commercial use, distribution, and reproduction in any medium, provided the original work is properly cited. 
and rectal cancer [5].

Many studies have reported on the risk of second cancer after prostate cancer treatment while including men of all ages at the time of diagnosis [6-28]. These studies may underestimate the risk of second cancer due to under-reporting from limited follow-up for older patients. So far, no study only evaluates the risk of second cancer in men $\leq 65$ years of age. The purpose of this study is to report the risk of bladder and rectal/rectosigmoid junction (RJ) cancer, using the risk of lung cancer as a reference, after prostate cancer diagnosis among this younger cohort. This information is much-needed for cancer treatment consent when discussing options at the time of prostate cancer diagnosis, and for guidance for second cancer screening for young men diagnosed with prostate cancer.

\section{Materials and Methods}

Eligible patients $(n=150,915)$ were identified in the 1973-2014 multi-primary SEER (Surveillance, Epidemiology, and End Results) database. Patients $\leq 65$ years of age at diagnosis with prostate cancer as their first malignancy were included. Patients were excluded from further analysis if they were diagnosed by autopsy or death certificate; had second cancer diagnosis less than 12 months following a prostate cancer diagnosis; had less than 12 months of follow-up after prostate cancer diagnosis; were listed as having received a form of radiation therapy that was not BEAM, SEED, or a combination of both; or received post-operative radiation treatment after radical prostatectomy. Selected patients were separated into four categories based on treatment received for primary prostate cancer: 92,679 underwent surgery without any form of radiation listed, 36,225 underwent BEAM alone, 13,001 underwent SEED alone, and 9,010 underwent both BEAM and SEED.

We identified the urinary bladder and the rectum/RJ as organs of interest in which to monitor second cancer events. These organs were chosen due to their proximity to the prostate, thus being more likely to receive incidental radiation during therapy. We also monitored second lung cancer as a reference group.

\section{Statistical analysis}

Descriptive statistics characterizing the study cohort were generated and compared between treatment modality groups. Continuous variables were compared using ANOVA, and categorical variables were compared using the chi-square test. To account for varying lengths of follow-up, we calculated the incidence rates (IR) of any second cancer, and specific second diagnoses of bladder, rectum/RJ, and lung cancers, for each treatment modality. Incidence rates were calculated as the number of observed second cancers over the number of person-years at risk (reported per 100 person-years). Person-years at risk were defined as the time from 12 months post-prostate cancer diagnosis to second cancer diagnosis of any type, death, or end of follow-up, whichever occurred first. Subjects with less than 12 months of follow-up post-prostate cancer diagnosis were excluded, and second events with a latency of less than 12 months were discarded to avoid including occurrences of cancers that likely antedated the treatment for prostate cancer. With an offset of person-years at risk, Poisson regression was used to generate incidence rate ratios comparing the risk of second cancer between treatment modalities. Multivariate Poisson models were generated to adjust for potential confounders, including age at primary prostate cancer diagnosis, race, and year of primary prostate cancer diagnosis. Cumulative incidence functions were used to graphically summarize the risk of any second cancer diagnosis and specific cancer diagnosis of interest over time, by treatment modality, in order to account for the presence of many competing risks. For the lung, bladder, and rectum/RJ cumulative incidence analyses, subjects with a subsequent malignancy of any other type were censored at the time of the second malignancy. Subjects alive and free from subsequent malignancy throughout available follow-up were censored at the date of the last follow-up. The Kaplan-Meier method was used to graphically summarize overall survival from the time of primary prostate cancer diagnosis to death from any cause; and was also used to graphically summarize second cancer-free survival from 12 months post-primary prostate cancer diagnosis to second malignancy or death, whichever occurred first. SAS 9.2 (SAS Institute Inc., Cary, NC, USA) was used for all analyses.

\section{Results}

Patient characteristics can be seen in Table 1. There were significant differences in age, year of primary cancer diagnosis, and race among patients treated with surgery, BEAM, SEED, and combined radiation.

The incidence rate per 100 person-years, incidence rate ratio (IRR), and IRR adjusted for race, age at primary prostate cancer diagnosis, and year of primary prostate cancer diagnosis are reported in Table 2 (detailed information regarding statistical analysis is included in Supplement A, B). The adjusted IRRs for any second malignancy and lung cancer after treatment for BEAM, SEED, and combined radiation, compared with surgery, were significantly higher (Table 2). The adjusted IRRs for bladder cancer after treatment for BEAM, SEED, and combined radiation, compared with surgery, were 2.09 (95\% confidence interval $[\mathrm{Cl}], 1.88-2.32), 1.91$ (95\% Cl, 1.61-2.26), and 2.04 (95\% Cl, 1.70-2.45), respectively. The 
Table 1. Study patient characteristics

\begin{tabular}{|c|c|c|c|c|c|}
\hline & Surgery $(n=92,679)$ & BEAM $(n=36,225)$ & SEED $(n=13,001)$ & Combined $(n=9,010)$ & $p$-value \\
\hline Person-years at risk & $886,757.2$ & 297,935 & $99,396.6$ & $75,740.1$ & \\
\hline Age at prostate cancer diagnosis (yr) & $58.2 \pm 5.3$ & $59.7 \pm 4.7$ & $59.0 \pm 4.8$ & $58.8 \pm 4.9$ & $<0.0001$ \\
\hline Year of primary diagnosis & $2,000.5 \pm 9.2$ & $1,998.0 \pm 10.5$ & $2,004.8 \pm 4.8$ & $2,002.9 \pm 6.6$ & $<0.0001$ \\
\hline Race & & & & & $<0.0001$ \\
\hline White & $77,443(83.6)$ & $26,947(74.4)$ & $10,356(79.7)$ & $6,288(69.8)$ & \\
\hline Black & $11,213(12.1)$ & $7,246(20.0)$ & $1,962(15.1)$ & $2,318(25.7)$ & \\
\hline Other & $3,218(3.5)$ & $1,755(4.8)$ & $563(4.3)$ & $353(3.9)$ & \\
\hline Unknown & $805(0.9)$ & $277(0.8)$ & $120(0.9)$ & $51(0.6)$ & \\
\hline SEER historic stage $A$ & & & & & $<0.0001$ \\
\hline Localized/regional & 70,615 (76.2) & $22,209(61.3)$ & $12,594(96.9)$ & 8,167 (90.6) & \\
\hline Un-staged & $489(0.5)$ & $234(0.7)$ & $102(0.8)$ & $88(1.0)$ & \\
\hline Distant & $96(0.1)$ & $1003(2.8)$ & $19(0.2)$ & $39(0.4)$ & \\
\hline Blank & $21,479(23.2)$ & $12,779(35.3)$ & $286(2.2)$ & $716(8.0)$ & \\
\hline Any second cancer diagnosis & $9,796(10.6)$ & 4,807 (13.3) & $1,298(10.0)$ & $1,018(11.3)$ & \\
\hline Lung & $1,620(1.8)$ & $1,000(2.8)$ & $186(1.4)$ & $166(1.8)$ & \\
\hline Bladder & $828(0.9)$ & $625(1.7)$ & $170(1.3)$ & $137(1.5)$ & \\
\hline Rectum/RJ & $370(0.4)$ & $219(0.6)$ & $49(0.4)$ & $59(0.7)$ & \\
\hline Age at second cancer diagnosis & $69.2 \pm 7.4$ & $69.3 \pm 7.1$ & $67.1 \pm 5.9$ & $67.5 \pm 6.3$ & $<0.0001$ \\
\hline Latency of second cancer diagnosis (yr) & $9.7 \pm 6.0$ & $8.8 \pm 5.9$ & $7.0 \pm 4.2$ & $7.6 \pm 4.9$ & \\
\hline
\end{tabular}

Values are presented as mean \pm standard deviation or number (\%).

BEAM, external beam radiation; SEED, brachytherapy, SEER, Surveillance, Epidemiology, and End Results; RJ, rectosigmoid junction.

Table 2. Incidence rate and ratio (IRR) with adjustment for age, year of primary prostate cancer diagnosis, and race

\begin{tabular}{|c|c|c|c|c|}
\hline & Total person-years & $\begin{array}{l}\text { Incidence rate per } 100 \text { person-years } \\
\qquad(95 \% \mathrm{Cl})\end{array}$ & $\operatorname{IRR}(95 \% \mathrm{Cl})$ & Adjusted IRR ${ }^{\text {a) }}(95 \% \mathrm{Cl})$ \\
\hline \multicolumn{5}{|c|}{ Modelling any second malignancy } \\
\hline Surgery & $886,757.2$ & $1.10(1.08,1.13)$ & 1.0 (ref) & 1.0 (ref) \\
\hline BEAM & 297,935 & $1.61(1.57,1.66)$ & $1.46(1.41,1.51)^{*}$ & $1.35(1.30,1.40)^{*}$ \\
\hline SEED & $99,396.6$ & $1.31(1.24,1.38)$ & $1.18(1.12,1.25)^{*}$ & $1.20(1.13,1.27)^{*}$ \\
\hline Combined & $75,740.1$ & $1.34(1.26,1.43)$ & $1.22(1.14,1.30)^{*}$ & $1.22(1.14,1.30)^{*}$ \\
\hline \multicolumn{5}{|c|}{ Modelling lung second malignancy } \\
\hline Surgery & $886,757.2$ & $0.18(0.17,0.19)$ & 1.0 (ref) & 1.0 (ref) \\
\hline BEAM & 297,935 & $0.34(0.32,0.36)$ & $1.84(1.70,1.99)^{*}$ & $1.60(1.48,1.74)^{*}$ \\
\hline SEED & $99,396.6$ & $0.19(0.16,0.22)$ & $1.02(0.88,1.19)$ & $1.11(0.95,1.30)$ \\
\hline Combined & $75,740.1$ & $0.22(0.19,0.26)$ & $1.20(1.02,1.41)^{*}$ & $1.22(1.03,1.43)^{*}$ \\
\hline \multicolumn{5}{|c|}{ Modelling bladder second malignancy } \\
\hline Surgery & $886,757.2$ & $0.09(0.087,0.10)$ & 1.0 (ref) & 1.0 (ref) \\
\hline BEAM & 297,935 & $0.21(0.19,0.23)$ & $2.25(2.03,2.49)^{*}$ & $2.09(1.88,2.32)^{*}$ \\
\hline SEED & $99,396.6$ & $0.17(0.15,0.20)$ & $1.83(1.55,2.16)^{*}$ & $1.91(1.61,2.26)^{*}$ \\
\hline Combined & $75,740.1$ & $0.18(0.15,0.21)$ & $1.94(1.62,2.32)^{*}$ & $2.04(1.70,2.45)^{*}$ \\
\hline \multicolumn{5}{|c|}{ Modelling rectum/RJ second malignancy } \\
\hline Surgery & $886,757.2$ & $0.04(0.04,0.05)$ & 1.0 (ref) & 1.0 (ref) \\
\hline BEAM & 297,935 & $0.07(0.06,0.08)$ & $1.76(1.49,2.08)^{*}$ & $1.58(1.33,1.87)^{*}$ \\
\hline SEED & $99,396.6$ & $0.05(0.04,0.07)$ & $1.18(0.88,1.59)$ & $1.30(0.96,1.76)$ \\
\hline Combined & $75,740.1$ & $0.08(0.06,0.10)$ & $1.87(1.42,2.46)^{*}$ & $1.98(1.50,2.61)^{*}$ \\
\hline
\end{tabular}

BEAM, external beam radiation; SEED, brachytherapy; $\mathrm{RJ}$, rectosigmoid junction.

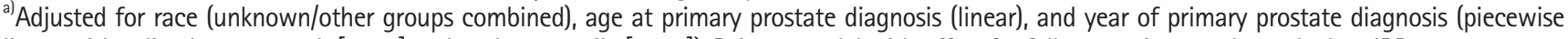
linear with spline knots at 25th [1995] and 75th percentile [2008]). Poisson model with offset for follow-up time used to calculate IRRs.

*Indicates statistical significance at alpha $=0.05$ level. 
adjusted IRRs for rectum/RJ cancer after treatment for BEAM, SEED, and combined radiation, compared with surgery, were 1.58 (95\% Cl, 1.33-1.87), $1.30(95 \% \mathrm{Cl}, 0.96-1.76)$, and $1.98(95 \% \mathrm{Cl}$, 1.50-2.61), respectively. The adjusted IRRs for lung cancer after treatment for BEAM, SEED, and combined radiation, compared with surgery, were 1.60 (95\% Cl, 1.48-1.74), 1.11 (95\% Cl, 0.95-1.30), and 1.22 (95\% Cl, 1.03-1.43), respectively.

The cumulative incidence of second cancer is depicted in Fig. 1 (detailed statistical analysis provided in Supplement C). When comparing all second cancers' cumulative incidences, there were noticeable differences between men treated with surgery and any form of radiation (Fig. 1A). Cumulative incidences of second lung, bladder, and rectum/RJ cancer were higher in men treated with any form of radiation than in men treated with surgery (Fig. 1A, 1C, and $1 D$, respectively). Cumulative incidences of second lung, bladder, and rectum/RJ cancer at 5 years after prostate cancer diagnosis are listed in Table 3. At 5 years after prostate cancer diagnosis, cumulative incidences of lung and bladder cancer were different
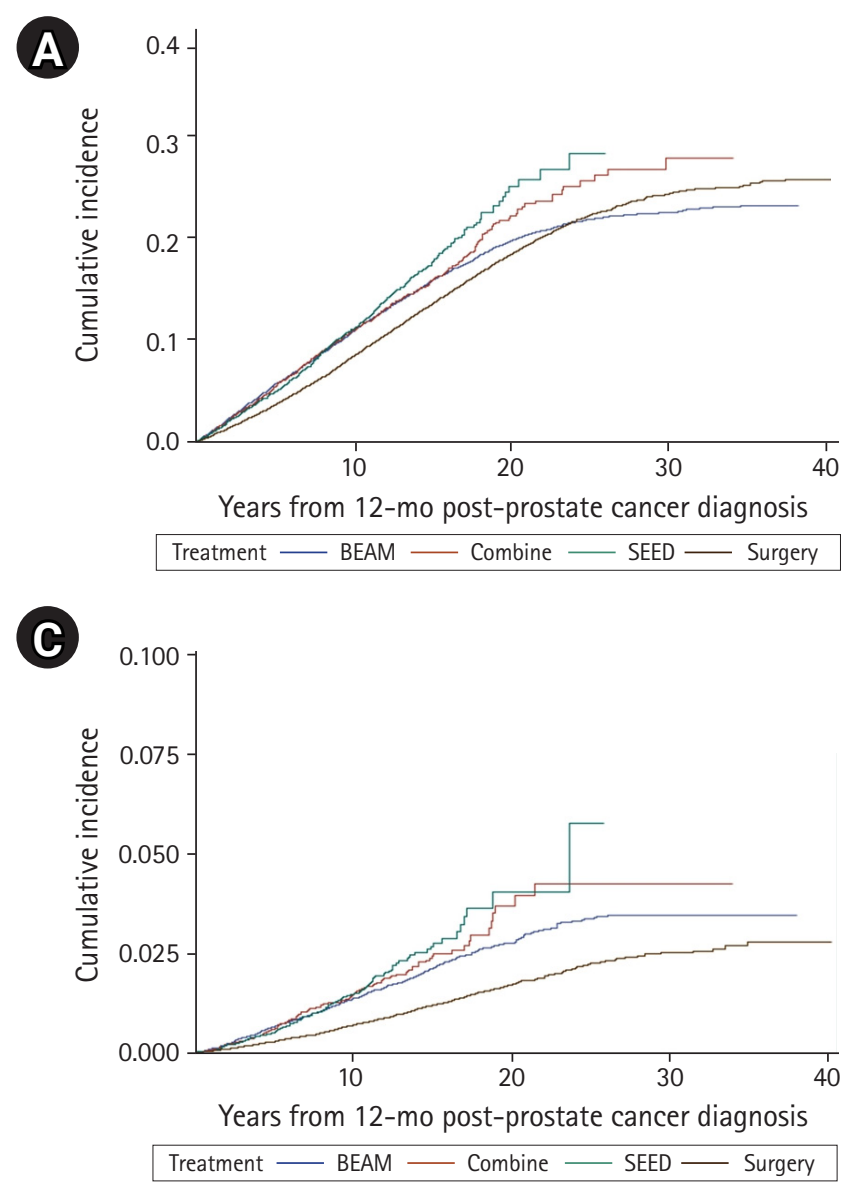

between men treated with surgery and any forms of radiation.

Overall survival and second cancer-free survival are shown in Fig. 2. Men treated with BEAM had worse overall survival and second cancer-free survival.

\section{Discussion and Conclusion}

Radiation-induced secondary cancer is generally considered to arise 5 years or more after receiving radiation, and to be located within the radiation field, to be a different histological type of primary cancer, and to not be present at the time of radiation treatment [29]. We observed that differences in the cumulative incidence of second bladder cancers were apparent between men treated with surgery and men treated with either form of radiation at 5 years of diagnosis. There was a higher incidence of lung cancer for men treated with either form of radiation, compared to men treated with surgery. These observations suggest that men elected to receive radiation might have other health-related factors con-

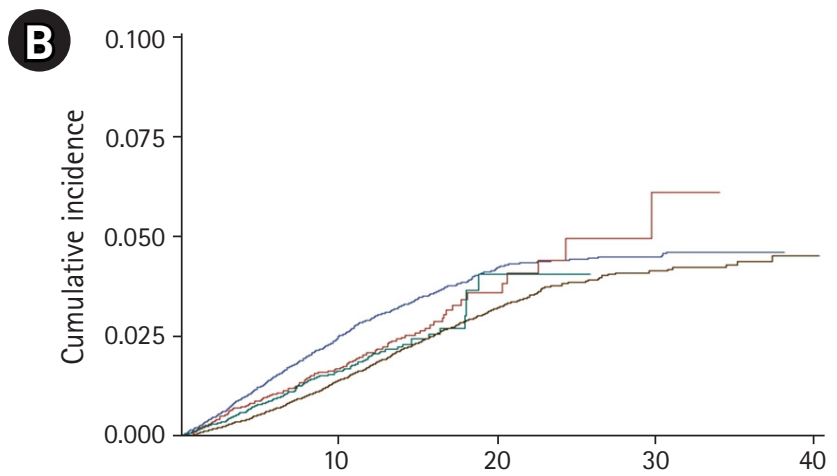

Years from 12-mo post-prostate cancer diagnosis

Treatment - BEAM - Combine - SEED — Surgery

D)

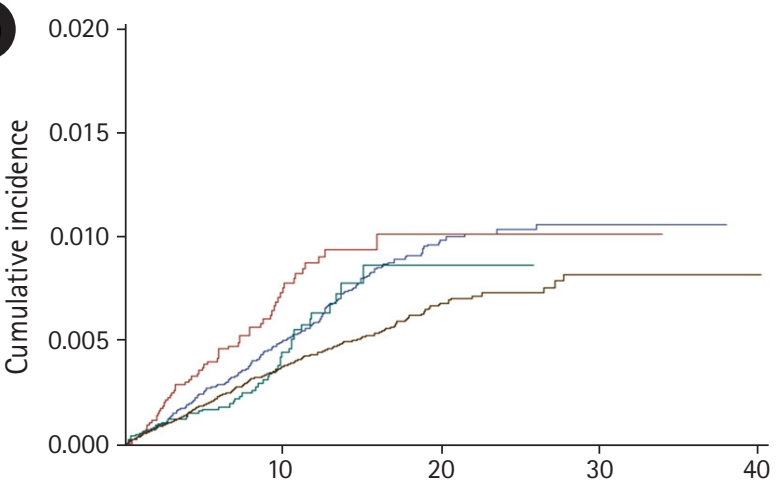

Years from 12-mo post-prostate cancer diagnosis Treatment $\longrightarrow$ BEAM - Combine - SEED — Surgery

Fig. 1. Cumulative incidence of second cancer from 12-month post-prostate cancer diagnosis. (A) Any second cancer. (B) Second lung cancer. (C) Second bladder cancer. (D) Second rectum/RJ cancer. BEAM, external beam radiation; SEED, brachytherapy; RJ, rectosigmoid junction. 
tributing to second cancer development. This hypothesis was also supported by the findings of Hegemann et al. [23] after their review of second cancer incidence after radiation, radiation after surgery, and surgery alone in a population-based clinical cancer

Table 3. Cumulative incidence of second cancer at 5 years after prostate cancer diagnosis

\begin{tabular}{|c|c|c|}
\hline & Cumulative incidence (\%) & $95 \% \mathrm{Cl}$ \\
\hline \multicolumn{3}{|c|}{ Any second malignancy } \\
\hline Surgery & 3.01 & $2.90-3.13$ \\
\hline BEAM & 4.73 & $4.50-4.96$ \\
\hline Combine & 4.46 & $4.03-4.92$ \\
\hline SEED & 4.25 & $3.89-4.62$ \\
\hline \multicolumn{3}{|l|}{ Lung } \\
\hline Surgery & 0.44 & $0.40-0.48$ \\
\hline BEAM & 1.01 & $0.90-1.12$ \\
\hline Combine & 0.78 & $0.61-0.99$ \\
\hline SEED & 0.65 & $0.52-0.81$ \\
\hline \multicolumn{3}{|l|}{ Bladder } \\
\hline Surgery & 0.22 & $0.19-0.26$ \\
\hline BEAM & 0.51 & $0.43-0.59$ \\
\hline Combine & 0.41 & $0.29-0.57$ \\
\hline SEED & 0.42 & $0.31-0.55$ \\
\hline \multicolumn{3}{|l|}{ Rectum/RJ } \\
\hline Surgery & 0.16 & $0.13-0.18$ \\
\hline BEAM & 0.20 & $0.16-0.25$ \\
\hline Combine & 0.31 & $0.21-0.46$ \\
\hline SEED & 0.15 & $0.09-0.23$ \\
\hline
\end{tabular}

BEAM, external beam radiation; SEED, brachytherapy; RJ, rectosigmoid junction; $\mathrm{Cl}$, confidence interval.

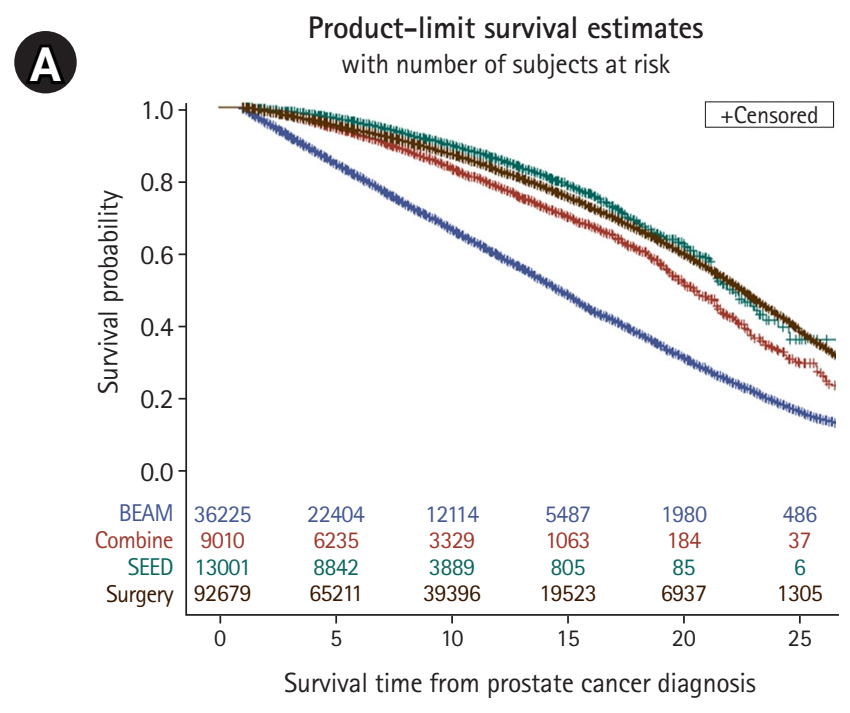

$$
\text { Treatment — BEAM — Combine — SEED — Surgery }
$$

registry in Bavaria. The authors suggested that a higher incidence of second cancer after radiation alone is likely related to advanced age and lifestyle habits.

Our study observed that second lung cancer rates were significantly elevated in men receiving BEAM or combined radiation compared to men treated with surgery, and others have also noted similar findings [24]. This is intriguing in part because lung tissue is not expected to receive radiation doses during radiation treatment regardless of treatment modality to the pelvis. On the other hand, smoking is a known risk factor for lung cancer. Smokers may have higher rates of respiratory insufficiency that result in increased risks when subject to general anesthesia, thus making them more likely to receive radiation as opposed to surgery. In fact, the incidence rates of primary lung cancer in the general population of men $\leq 65$ years is 0.28 per 100 person-years [1], compared with 0.18 and 0.34 per 100 person-years for men treated with surgery and BEAM respectively. Furthermore, smoking is a risk factor for bladder cancer, and a difference in smoking rates between treatment groups may partially explain the increased second bladder cancer risk in patients receiving radiation therapy. The incidence rates of primary bladder cancer in men younger than 65 years of age were 0.10 per 100 person-years, as reported in the SEER database [1]. In our study cohort, the incidence rates of bladder cancer in men treated with BEAM, SEED, and combined radiation were $0.21,0.17$, and 0.18 per 100 person-years comparing with 0.09 per 100 person-years in men treated with surgery. The fact that increased incidence of bladder cancer was observed 5 years post-prostate cancer diagnosis also suggested that radiation may

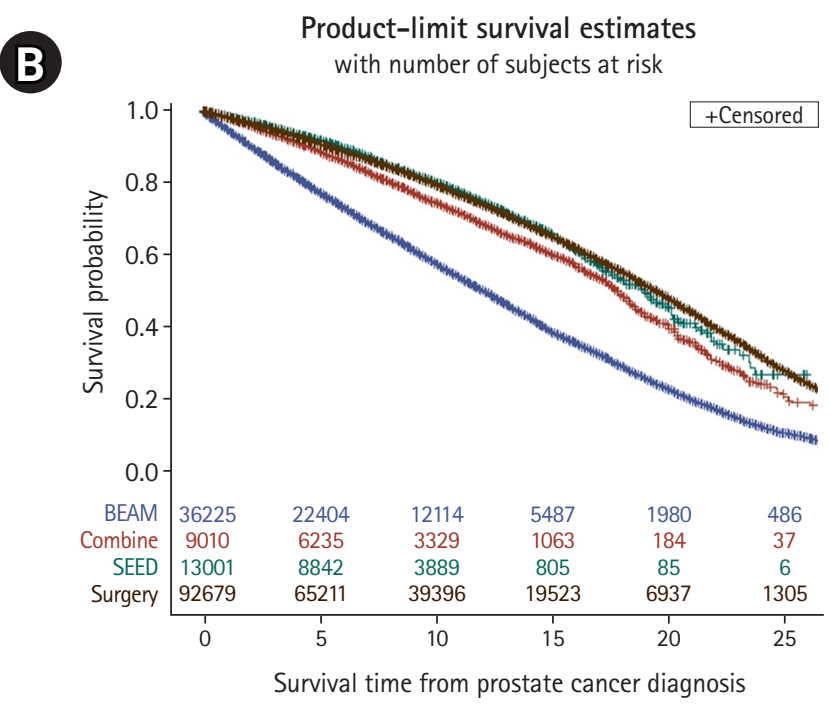

Treatment — BEAM — Combine — SEED — Surgery

Fig. 2. Overall survival and second cancer-free survival. (A) Overall survival after first prostate cancer diagnosis. (B) Second cancer-free survival (over time at risk). BEAM, external beam radiation; SEED, brachytherapy. 
not be the only risk factor for second bladder cancer. Unfortunately, the SEER database lacks detailed information regarding predisposing factors, including smoking status or chronic lung disease. Therefore, we cannot validate this hypothesis in our current study.

Incidence rates of primary rectum/RJ cancer in men younger than 65 years of age were 0.08 per 100 person-years, as reported in the SEER database [1]. In our study cohort, the incidence rates of rectum/RJ cancer in men treated with surgery, BEAM, SEED, and combined radiation were $0.04,0.07$, and 0.05 per 100 person-years, respectively. The relatively low incidence rate of second rectum/RJ cancer in our control cohort (men treated with surgery for prostate cancer) compared with the incidence rate of rectum/RJ cancer as a primary diagnosis in the general population might be related to intrinsic limitations of data reporting or other factors beyond the scope of this investigation. Further, incidences of rectum/RJ cancer after any form of radiation were comparable to the incidence in the general population, suggesting that the increase in incidence in men treated with radiation might be exaggerated when comparison was made with the incidence after surgery.

Regardless of the cause of second cancers, our observation reflects the status of second cancer risk in men younger than 65 treated with some form of radiation as reported to the SEER database. There was about 100\% increased risk of second bladder cancer in men receiving radiation compared with men treated with surgery. The risk of second bladder cancer observed in our study was higher than in published series including men of all ages. Brenner et al. [26] reported an increased risk of bladder cancer and rectal cancer (77\% and $105 \%$ at 5 and 10 years, respectively) after radiation compared to surgery after reviewing the SEER database. At the time of prostate cancer diagnosis, the median ages of men were 70.3 years for men treated with radiation and 71.4 years for men treated with surgery, with $<10 \%$ of men from either group $<60$ year of age. Nieder et al. [27] reported that the relative risk of bladder cancer after external beam radiotherapy, brachytherapy, and combined radiation after more than 10 years of follow-up compared to radical prostatectomy was $1.83,0.47$, and 1.64 , respectively, as reported to the SEER database. Moon et al. [19] reported statistically significant increased odds of bladder cancer (odds ratio $[\mathrm{OR}]=1.63 ; 95 \% \mathrm{Cl}, 1.44-1.84$ ) after external beam radiation for prostate cancer, compared with men who received no prostate cancer-directed radiation. After reviewing the Cancer of the Prostate Strategic Urologic Research Endeavor (CaPSURE) disease registry, Boorjian et al. [28] reported patients treated with radical prostatectomy were approximately half as likely to have post-treatment bladder cancer as patients who underwent radiation therapy (hazard ratio $=0.51 ; 95 \% \mathrm{Cl}, 0.29-0.89$ ). After reviewing a large French-Canadian population-based cohort of pros- tate cancer patients, Bhojani et al. [30] reported 40\% increased incidence (hazard ratio $=1.4 ; p=0.02$ ) of bladder cancer for men post-radiation for prostate cancer compared to men post-surgery.

Our study has several weaknesses due to the retrospective design and inherent deficiencies of data reported to the SEER program. There was no information about patients' performance status, medical co-morbidities, or family history of cancer in the SEER database. These factors might have influenced the choice of prostate cancer treatment and, therefore, second cancer risk. Additionally, there are potential issues of under-reporting of second cancers, especially in elderly patients, to the SEER database. Our study population, comprised of men $\leq 65$ years of age when diagnosed with prostate cancer, may mitigate this risk.

Despite the above-stated weaknesses, our observations, together with others, underscore the challenges prostate cancer survivors face, in the years after prostate cancer diagnosis. Higher risks of any second malignancy, including lung, bladder, and rectal/RJ cancers, among young men treated with radiation for prostate cancer compared with surgery, regardless of underlying causes, suggests that early and continuous vigorous second cancer screening practice may be beneficial for younger prostate cancer survivors.

In conclusion, compared to men treated with surgery, younger prostate cancer survivors had an increased risk of second malignancy, including lung, bladder, and rectum/RJ cancer, after treatment with external beam or combined radiation. The cause of the observed increase in second cancer risk in men treated with radiation is likely multifactorial. Although the true extent of increase in second cancer risk (specifically second rectum/RJ cancer) after radiation requires further investigation, second cancer screening should be an essential aspect of post-treatment survivorship care plan for young prostate cancer survivors.

\section{Conflict of Interest}

No potential conflict of interest relevant to this article was reported.

\section{Acknowledgements}

We thank Mrs. Laura Finger for her excellent editorial and administrative assistance.

\section{Supplementary Materials}

Supplementary materials can be found via https://doi.org/10.3857/ roj.2020.00857. 


\section{References}

1. American Cancer Society. Key Statistics for prostate cancer. Atlanta, GA: American Cancer Society; c2021 [cited 2021 May 30]. Available from: https://www.cancer.org/cancer/prostate-cancer/ about/key-statistics.html.

2. Resnick MJ, Lacchetti C, Penson DF; American Society of Clinical Oncology. Prostate cancer survivorship care guidelines: American Society of Clinical Oncology practice guideline endorsement. J Oncol Pract 2015;11:e445-9.

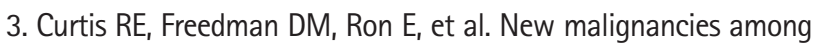
cancer survivors: SEER Cancer Registries, 1973-2000 (NIH Publ. No. 05-5302) Bethesda, MD: National Cancer Institute; 2006.

4. Donin N, Filson C, Drakaki A, et al. Risk of second primary malignancies among cancer survivors in the United States, 1992 through 2008. Cancer 2016;122:3075-86.

5. Dracham CB, Shankar A, Madan R. Radiation induced secondary malignancies: a review article. Radiat Oncol J 2018;36:85-94.

6. Wallis CJ, Mahar AL, Choo R, et al. Second malignancies after radiotherapy for prostate cancer: systematic review and meta-analysis. BMJ 2016;352:i851.

7. Abdel-Wahab M, Reis IM, Hamilton K. Second primary cancer after radiotherapy for prostate cancer: a seer analysis of brachytherapy versus external beam radiotherapy. Int J Radiat Oncol Biol Phys 2008;72:58-68.

8. Abern MR, Dude AM, Tsivian M, Coogan CL. The characteristics of bladder cancer after radiotherapy for prostate cancer. Urol Oncol 2013;31:1628-34.

9. Baxter NN, Tepper JE, Durham SB, Rothenberger DA, Virnig BA. Increased risk of rectal cancer after prostate radiation: a population-based study. Gastroenterology 2005;128:819-24.

10. Berrington de Gonzalez A, Curtis RE, Kry SF, et al. Proportion of second cancers attributable to radiotherapy treatment in adults: a cohort study in the US SEER cancer registries. Lancet Oncol 2011;12:353-60.

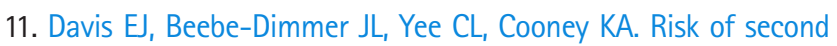
primary tumors in men diagnosed with prostate cancer: a population-based cohort study. Cancer 2014;120:2735-41.

12. Zelefsky MJ, Pei $X$, Teslova T, et al. Secondary cancers after intensity-modulated radiotherapy, brachytherapy and radical prostatectomy for the treatment of prostate cancer: incidence and cause- specific survival outcomes according to the initial treatment intervention. BJU Int 2012;110:1696-701.

13. Pickles T, Phillips N. The risk of second malignancy in men with prostate cancer treated with or without radiation in British Columbia, 1984-2000. Radiother Oncol 2002;65:145-51.

14. Margel D, Baniel J, Wasserberg N, Bar-Chana M, Yossepowitch 0.
Radiation therapy for prostate cancer increases the risk of subsequent rectal cancer. Ann Surg 2011;254:947-50.

15. Huang J, Kestin LL, Ye $H$, Wallace M, Martinez AA, Vicini FA. Analysis of second malignancies after modern radiotherapy versus prostatectomy for localized prostate cancer. Radiother Oncol 2011;98:81-6.

16. Singh A, Kinoshita Y, Rovito PM Jr, et al. Higher than expected association of clinical prostate and bladder cancers. J Urol 2008;179(5 Suppl):S2-5.

17. Hinnen KA, Schaapveld $M$, van Vulpen $M$, et al. Prostate brachytherapy and second primary cancer risk: a competitive risk analysis. J Clin Oncol 2011;29:4510-5.

18. Huo D, Hetzel JT, Roy H, Rubin DT. Association of colorectal cancer and prostate cancer and impact of radiation therapy. Cancer Epidemiol Biomarkers Prev 2009;18:1979-85.

19. Moon K, Stukenborg GJ, Keim J, Theodorescu D. Cancer incidence after localized therapy for prostate cancer. Cancer 2006;107: 991-8.

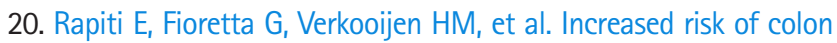
cancer after external radiation therapy for prostate cancer. Int J Cancer 2008;123:1141-5.

21. Singh AK, Mashtare TL, McCloskey SA, Seixas-Mikelus SA, Kim $\mathrm{HL}$, May KS. Increasing age and treatment modality are predictors for subsequent diagnosis of bladder cancer following prostate cancer diagnosis. Int J Radiat Oncol Biol Phys 2010;78: 1086-94.

22. Vuolukka K, Auvinen P, Palmgren JE, Aaltomaa S, Kataja V. Incidence of subsequent primary cancers and radiation-induced subsequent primary cancers after low dose-rate brachytherapy monotherapy for prostate cancer in long-term follow-up. BMC Cancer 2020;20:453.

23. Hegemann NS, Schlesinger-Raab A, Ganswindt $U$, et al. Risk of second cancer following radiotherapy for prostate cancer: a population-based analysis. Radiat Oncol 2017;12:2.

24. Sountoulides P, Koletsas N, Kikidakis D, Paschalidis K, Sofikitis N. Secondary malignancies following radiotherapy for prostate cancer. Ther Adv Urol 2010;2:119-25.

25. Murray L, Henry A, Hoskin P, Siebert FA, Venselaar J; PROBATE group of GEC ESTRO. Second primary cancers after radiation for prostate cancer: a systematic review of the clinical data and impact of treatment technique. Radiother Oncol 2014;110:213-28.

26. Brenner DJ, Curtis RE, Hall EJ, Ron E. Second malignancies in prostate carcinoma patients after radiotherapy compared with surgery. Cancer 2000;88:398-406.

27. Nieder AM, Porter MP, Soloway MS. Radiation therapy for prostate cancer increases subsequent risk of bladder and rectal cancer: a population based cohort study. J Urol 2008;180:2005-9. 
28. Boorjian S, Cowan JE, Konety BR, et al. Bladder cancer incidence and risk factors in men with prostate cancer: results from Cancer of the Prostate Strategic Urologic Research Endeavor. J Urol 2007;177:883-7.

29. Li Cl, Nishi N, McDougall JA, et al. Relationship between radiation exposure and risk of second primary cancers among atomic bomb survivors. Cancer Res 2010;70:7187-98.

30. Bhojani N, Capitanio U, Suardi N, et al. The rate of secondary malignancies after radical prostatectomy versus external beam radiation therapy for localized prostate cancer: a population-based study on 17,845 patients. Int J Radiat Oncol Biol Phys 2010;76: 342-8. 